University of Nebraska - Lincoln

DigitalCommons@University of Nebraska - Lincoln

Timothy J. Gay Publications

Research Papers in Physics and Astronomy

$3-25-2002$

\title{
Effect of Relativistic Many-Electron Interactions on Photoelectron Partial Wave Probabilities
}

\author{
K. W. McLaughlin \\ Loras College, Dubuque, lowa \\ Orhan Yenen \\ University of Nebraska - Lincoln, oyenen1@unl.edu \\ Duane H. Jaecks \\ University of Nebraska-Lincoln, djaecks@unl.edu \\ Timothy J. Gay \\ University of Nebraska - Lincoln, tgay1@unl.edu \\ M. M. Sant'Anna \\ University of California, Berkeley, California \\ See next page for additional authors
}

Follow this and additional works at: https://digitalcommons.unl.edu/physicsgay

Part of the Physics Commons

McLaughlin, K. W.; Yenen, Orhan; Jaecks, Duane H.; Gay, Timothy J.; Sant'Anna, M. M.; Calabrese, D.; and Thaden-Jordan, B., "Effect of Relativistic Many-Electron Interactions on Photoelectron Partial Wave Probabilities" (2002). Timothy J. Gay Publications. 34.

https://digitalcommons.unl.edu/physicsgay/34

This Article is brought to you for free and open access by the Research Papers in Physics and Astronomy at DigitalCommons@University of Nebraska - Lincoln. It has been accepted for inclusion in Timothy J. Gay Publications by an authorized administrator of DigitalCommons@University of Nebraska - Lincoln. 


\section{Authors}

K. W. McLaughlin, Orhan Yenen, Duane H. Jaecks, Timothy J. Gay, M. M. Sant'Anna, D. Calabrese, and B. Thaden-Jordan 


\title{
Effect of Relativistic Many-Electron Interactions on Photoelectron Partial Wave Probabilities
}

\author{
K. W. McLaughlin, ${ }^{1}$ O. Yenen, ${ }^{2}$ D. H. Jaecks, ${ }^{2}$ T. J. Gay, ${ }^{2}$ M. M. Sant'Anna, ${ }^{3}$ D. Calabrese, ${ }^{4}$ and B. Thaden-Jordan ${ }^{2}$ \\ ${ }^{1}$ Department of Physics and Engineering, Loras College, Dubuque, Iowa 52004-0178 \\ ${ }^{2}$ Behlen Laboratory of Physics, University of Nebraska-Lincoln, Lincoln, Nebraska 68588-0111 \\ ${ }^{3}$ Advanced Light Source, Ernest Orlando Lawrence Berkeley National Laboratory, University of California, \\ Berkeley, California 94720 \\ ${ }^{4}$ Sierra Community College, Rocklin, California 95677 \\ (Received 22 October 2001; published 7 March 2002)
}

\begin{abstract}
We obtain relative cross sections for the production of photoelectrons with specific angular momentum quantum numbers. These cross sections are obtained from the polarization analysis of the visible fluorescence of ions produced when circularly polarized vacuum ultraviolet radiation photoionizes ground state Ar. The ratio of cross sections for the production of photoelectrons with the same orbital angular momentum but different total angular momenta shows strong deviations from the statistical ratio, demonstrating the importance of relativistic interactions in many-electron photoionization dynamics.
\end{abstract}

DOI: $10.1103 /$ PhysRevLett.88.123003

PACS numbers: $32.80 . \mathrm{Fb}$

An important goal in atomic physics is the articulation of the dynamics of multielectron processes. Photoionization is a uniquely useful tool in this regard, because it allows one to prepare atomic states with well-defined energies and angular momenta. Measurements of total and differential photoemission cross sections, photoelectron spin polarizations, and the fluorescence of the residual ions has elucidated a rich range of photoionization dynamics [1-7].

Recently, the surprising ubiquity of significant relativistic interactions during photoionization, where the spins and orbital angular momenta of the residual ionic constituents and/or the photoelectron couple, even in relatively light atoms such as $\mathrm{Ne}$ [6], has become apparent. These interactions manifest themselves through the production of quartet states in the residual excited ion of closed shell atoms [7-9], spin polarization of emitted electrons [10,11], and as we have shown in an earlier Letter [12], through the orientation of the ${ }^{2} P_{1 / 2}^{o}$ state of the residual $\mathrm{Ar}^{+}$.

In these processes, a complete determination of the partial wave probabilities of the photoelectron is especially important for a detailed understanding of relativistic dynamics. When relativistic interactions are negligible, the ratios of the partial wave probabilities for photoelectrons having the same orbital angular momentum $\ell_{e}$ but different total angular momentum $j_{e}$ are in the ratio of their statistical weights, i.e., their multiplicities. Deviations from these statistical ratios quantify the importance of relativistic interactions. However, to our knowledge, such studies of total angular momentum-resolved partial wave probabilities over an extended range of ionizing photon energies have not been carried out to date for multielectron processes. Snell et al. [13] determined the three partial wave probabilities of the photoelectron and their phase differences for fine-structure resolved $\mathrm{Xe} 4 d$ photoionization at a photon energy of $93.8 \mathrm{eV}$. Wang and Elliott [14] obtained the three partial wave probabilities of the photoelectron and their phase differences for the two-photon direct photoionization of atomic $\mathrm{Rb}$ in the range $0-0.5 \mathrm{eV}$ above the ionization threshold. Since Rb has only a single outer electron, the photoionization is a single electron process in their range of energies and proceeds through direct emission of the photoelectron.

In this Letter we report the results of a polarization analysis of the visible fluorescence from the excited residual ion following photoionization. From these measurements we have extracted the total-angular-momentum-resolved partial wave probabilities of the photoelectron over an extended range of photon energies. Our measurements highlight the significance of multielectron processes including the formation of autoionizing resonances. We have also obtained the expectation value of the $z$ component of the spin of the emitted electrons integrated over a $4 \pi$ solid angle.

The multielectron process we consider is the photoionization of Ar by circularly polarized VUV radiation from the threshold of formation of the excited residual ionic state $\operatorname{Ar}^{+}\left\{3 p^{4}\left[{ }^{3} P\right] 4 p^{2} P_{3 / 2}\right\}(35.627 \mathrm{eV}$ above the $\mathrm{Ar}$ ground state) to $36.650 \mathrm{eV}$. Figure 1 shows the schematic experimental setup. As previous measurements [15] with linearly polarized radiation have shown, the spectrum in this energy range is dominated by doubly excited resonances of Ar. We may write

$$
h \nu+\operatorname{Ar}\left\{3 p^{6}{ }^{1} S_{0}\right\} \rightarrow(\operatorname{Ar})^{* * *} \rightarrow \operatorname{Ar}^{+}\left\{3 p^{4}\left[{ }^{3} P\right] 4 p^{2} P_{3 / 2}^{o}\right\}+e^{-} \rightarrow \operatorname{Ar}^{+}\left\{3 p^{4}\left[{ }^{3} P\right] 4 s^{2} P_{1 / 2}\right\}+h \nu^{\prime}(476.5 \mathrm{~nm})+e^{-},
$$

where the triple stars on the intermediate resonances refer to the possibility of having doubly excited valence electrons in Ar with additional excitation in the coupling of the $3 p^{4}$ electrons.
The initial linearly polarized synchrotron radiation is transformed into $99.7 \%$ circularly polarized light using a four-reflector quarter-wave retarder we have installed on 


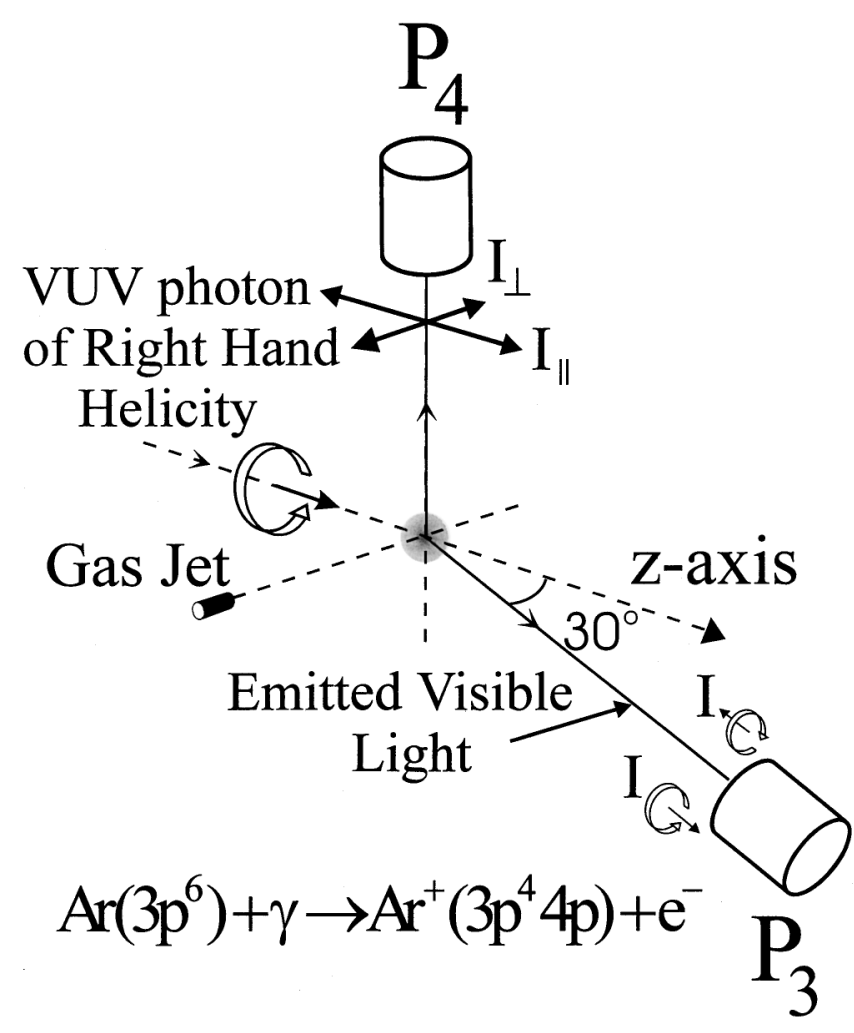

FIG. 1. Schematic of experimental setup. Circular polarization $\left(P_{3}\right)$ and linear polarization $\left(P_{4}\right)$ are measured as indicated. The quantization axis is taken to be parallel to the photon spin and thus flips direction when the incident photon's helicity is reversed.

beam line 10.0.2 of the Advanced Light Source (ALS) at the Lawrence Berkeley National Laboratory [16]. We measure the circular polarization of the fluorescent photons at $30^{\circ}$ with respect to the incident beam while simultaneously determining the linear polarization of the fluorescence emitted perpendicular to the incident beam for the same optical transition. The fluorescence at $476.5 \mathrm{~nm}$ is selected using two narrow-band interference filters $(\Delta \lambda=0.3 \mathrm{~nm})$ in front of the two detectors which allows us to resolve the fine-structure level of the excited residual ion and assign a total angular momentum $J=3 / 2$ to the ionic state.

When circularly polarized light of right-hand helicity is used, conservation of angular momentum requires that the final ion + photoelectron system must have a total angular momentum of $J_{t}=1$ with a projection of $M_{J_{t}}=+1$ onto the quantization axis, which is taken to be the incident beam direction. For the residual ionic state considered here, the total angular momentum $j_{e}$ of the photoelectron is limited to $1 / 2,3 / 2$, or $5 / 2$ by angular momentum and parity conservation laws. The orbital angular momentum $\ell_{e}$ of the photoelectron is similarly restricted to 0 or 2 ( $s$ or $d$ partial waves). For the $s$ waves $j_{e}=1 / 2$ and for $d$ waves $j_{e}=3 / 2$ or $5 / 2$. We denote the spin of the photoelectron by $s_{e}$.

Since there are several allowed $j_{e}$ values, the total wave function of the ion + photoelectron system has to be ex- pressed as a linear combination of angular momentum eigenstates for given $J$ and $J_{t}$ :

$$
|\psi\rangle=\sum_{j_{e}} a_{j_{e}}\left|J j_{e} J_{t} M_{J_{t}}\right\rangle
$$

where the partial wave probability amplitudes $a_{j_{e}}$ of the photoelectrons contain all the dynamical information about the photoionization process. As we show in this Letter, all three partial wave probabilities $\left|a_{j_{e}}\right|^{2}$ of the electrons emitted to a $4 \pi$ solid angle can be expressed in terms of the alignment and orientation parameters $A_{0}(J)$ and $O_{0}(J)$ of the excited residual ionic state that emits the fluorescence. We use the Fano-Macek formalism [17] to determine the alignment and orientation parameters from the measurements of linear polarization $P_{4}$ (the subscript 4 refers to the fact that the linear polarization is measured in the plane of symmetry of the system, i.e., in a direction perpendicular to the quantization axis) and circular polarization $P_{3}$.

The alignment and orientation parameters are defined in Ref. [17] as

$$
\begin{aligned}
A_{0}(J) & =\frac{\sum_{M_{J}}\left[3 M_{J}^{2}-J(J+1)\right]\left|\left\langle J M_{J} \mid \psi\right\rangle\right|^{2}}{J(J+1)} \\
\text { and } \quad O_{0}(J) & =\frac{\left\langle J_{z}\right\rangle}{J(J+1)}=\frac{\sum_{M_{J}} M_{J}\left|\left\langle J M_{J} \mid \psi\right\rangle\right|^{2}}{J(J+1)},
\end{aligned}
$$

where $|\psi\rangle$ represents the state of the electron + ion system and is given by Eq. (1). Moreover [17,18]

$$
\begin{aligned}
A_{0}(J) & =-\frac{4 P_{4}}{h^{(2)}\left(P_{4}-3\right)} \\
\text { and } \quad O_{0}(J) & =-\frac{2 P_{3}\left(P_{4} \cos ^{2} \theta-1\right)}{h^{(1)}\left(P_{4}-3\right) \cos \theta},
\end{aligned}
$$

where $\theta=30^{\circ}$ (Fig. 1) and $h^{(1)}$ and $h^{(2)}$ are constants that depend on the initial and final states of the fluorescent transition.

We note that the total angular momentum $\mathbf{J}_{t}$ of the ion + photoelectron system is shared by the residual ion and the photoelectron with total angular momenta $\mathbf{J}$ and $\mathbf{j}_{e}$, respectively; i.e., $\mathbf{J}_{t}=\mathbf{J}+\mathbf{j}_{e}$. Noting that $\mathbf{j}_{e}=\boldsymbol{\ell}_{e}+\mathbf{s}_{e}$, we can expand $|\psi\rangle$ in a Clebsch-Gordon series:

$$
\begin{aligned}
|\psi\rangle= & \sum_{j_{e}} a_{j_{e}} \sum_{\substack{M_{J} m_{j_{e}} \\
m_{\ell_{e}} m_{s_{e}}}}\left\langle J M_{J} j_{e} m_{j_{e}} \mid J_{t} M_{J_{t}}\right\rangle \\
& \times\left\langle\ell_{e} m_{\ell_{e}} s_{e} m_{s_{e}} \mid j_{e} m_{j_{e}}\right\rangle\left|J M_{J}\right\rangle\left|\ell_{e} m_{\ell_{e}}\right\rangle\left|s_{e} m_{s_{e}}\right\rangle .
\end{aligned}
$$

Projecting $|\psi\rangle$ onto $\left|J M_{J}\right\rangle$ we obtain $A_{0}(J)$ and $O_{0}(J)$ in terms of the partial wave probabilities $\left|a_{j_{e}}\right|^{2}$ :

$$
\begin{aligned}
\left|a_{1 / 2}\right|^{2} & =\frac{25 A_{0}(J)+30 O_{0}(J)+4}{24}, \\
\left|a_{3 / 2}\right|^{2} & =-\frac{5 A_{0}(J)-3 O_{0}(J)-1}{3}, \\
\text { and } \quad\left|a_{5 / 2}\right|^{2} & =\frac{5 A_{0}(J)-18 O_{0}(J)+4}{8} .
\end{aligned}
$$


In Fig. 2 we present the experimental measurements as a function of the ionizing photon energy: Fig. 2(a) shows the relative total intensity (polarization summed) of light observed at the top detector, perpendicular to the incident beam. The vertical bars represent the position of doubly excited Ar states obtained from known series limits and accepted ranges of quantum defects [19-23]. The complex structure of the fluorescent intensity is clearly correlated to the positions of the doubly excited Rydberg states of $\mathrm{Ar}^{* *}$. In the energy regions where only one or two $\mathrm{Ar}^{* * *}$ states are present, the fluorescent intensity exhibits a classic Fano profile. For example, the cross section is nearly zero at $36.146 \mathrm{eV}$ and a maximum at $36.164 \mathrm{eV}$ displaying the characteristic Fano profile shape. In the region of 35.84 to $36.15 \mathrm{eV}$ many levels of $\mathrm{Ar}^{* * *}$ are present and are interacting with the continuum to give a complex intensity spectrum.

For our photon energy range cascades from higher excited $\mathrm{Ar}^{+}$states are negligible. There are only four other excited states of $\mathrm{Ar}^{+}$within $1 \mathrm{eV}$ of the threshold

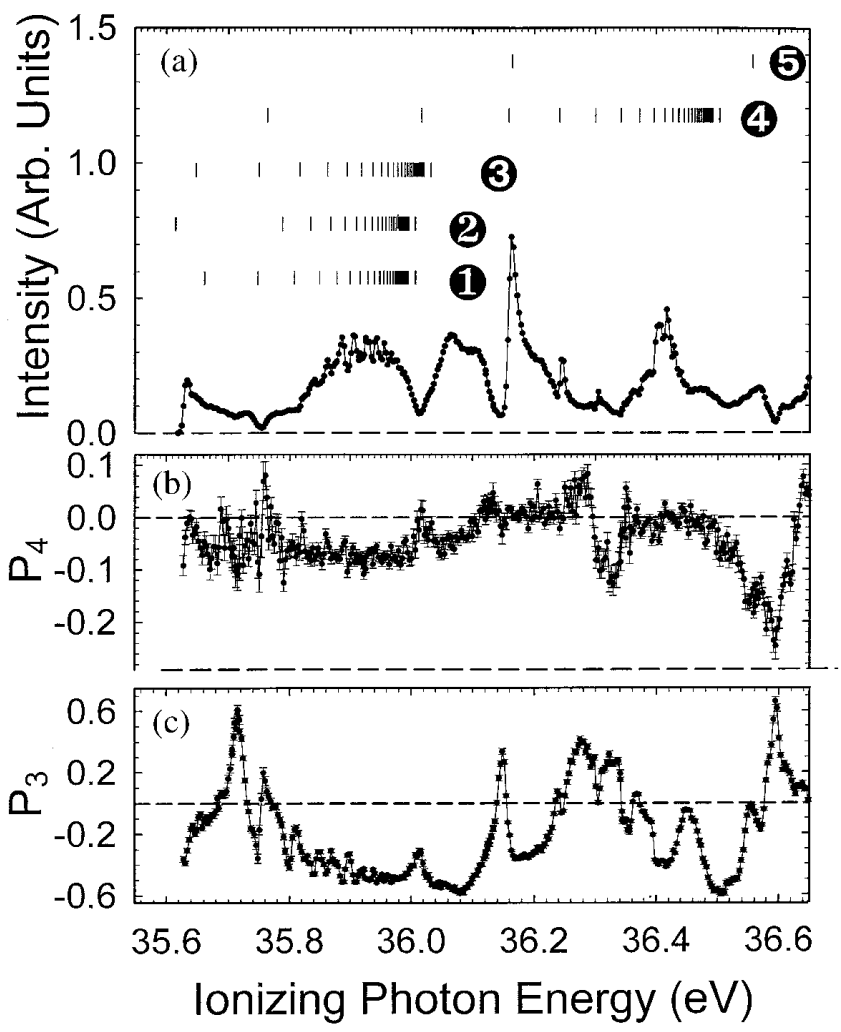

FIG. 2. (a) The relative total intensity of fluorescence observed perpendicular to the beam. The vertical lines show the known doubly excited states of Ar: (1) $3 p^{4}\left[{ }^{1} D\right] 3 d^{2} F_{5 / 2}$ $n p$ series starting with $n=8$, (2) $3 p^{4}\left[{ }^{1} D\right] 3 d^{2} F_{5 / 2} n f$ series starting with $n=6$, (3) $3 p^{4}\left[{ }^{1} D\right] 3 d^{2} F_{7 / 2} n f$ series starting with $n=6$, (4) $3 p^{4}\left[{ }^{1} S\right] 4 s^{2} S_{1 / 2} n p$ series starting with $n=6$, (5) $3 p^{4}\left[{ }^{1} D\right] 4 p^{2} P_{1 / 2} n s$ series starting with $n=6$; (b) linear polarization measured perpendicular to the beam; (c) circular polarization measured at $30^{\circ}$ with respect to the beam. Error bars represent 1 standard deviation in statistical uncertainty. The experimental resolution is about $8 \mathrm{meV}$. of formation of the state $\left(3 p^{4}\left[{ }^{3} P\right] 4 p^{2} P_{3 / 2}^{o}\right)$ that produce the fluorescence we observe. The $3 p^{4}\left[{ }^{3} P\right] 4 p^{2} S_{1 / 2}^{o}$ and $3 p^{4}\left[{ }^{3} P\right] 4 p^{4} S_{3 / 2}^{o}$ states are both odd and their decay to another odd state $\left(3 p^{4}\left[{ }^{3} P\right] 4 p^{2} P_{3 / 2}^{o}\right.$ in our case) is dipole forbidden. The states labeled $3 d^{\prime 2} F_{5 / 2,7 / 2}$ have ${ }^{1} D$ cores. Core-changing radiative decays (from ${ }^{1} D$ to ${ }^{3} P$ for our measurements) are again quite unlikely.

Figure 2(b) shows the linear polarization of the fluorescence measured perpendicular to the direction of the beam. Figure 2(c) is the circular polarization of the fluorescence measured at $30^{\circ}$ with respect to the initial beam direction as shown in Fig. 1.

Figure 3 shows the relative cross sections for the $s_{1 / 2}$, $d_{3 / 2}$, and $d_{5 / 2}$ partial waves of the photoelectron. They are determined by multiplying the total intensity times the partial wave probabilities obtained from the linear and circular polarization measurements using Eqs. (3) and (4). (Note that Fig. 3 does not include $0 \%-5 \%$ corrections to the total intensity due to the linear polarization variations.) The results indicate that Fano's argument [24] that $(\ell+1)$ partial waves (in our case $d$ partial waves) are preferred over $(\ell-1)$ partial waves ( $s$ partial waves) seems to be valid over most of the energy region studied. However, there are energy regions where this propensity fails, for reasons that are open to conjecture at this point.

Figure 4 shows the energy dependence of the ratio of the $d_{5 / 2}$ to $d_{3 / 2}$ cross sections. If relativistic interactions were

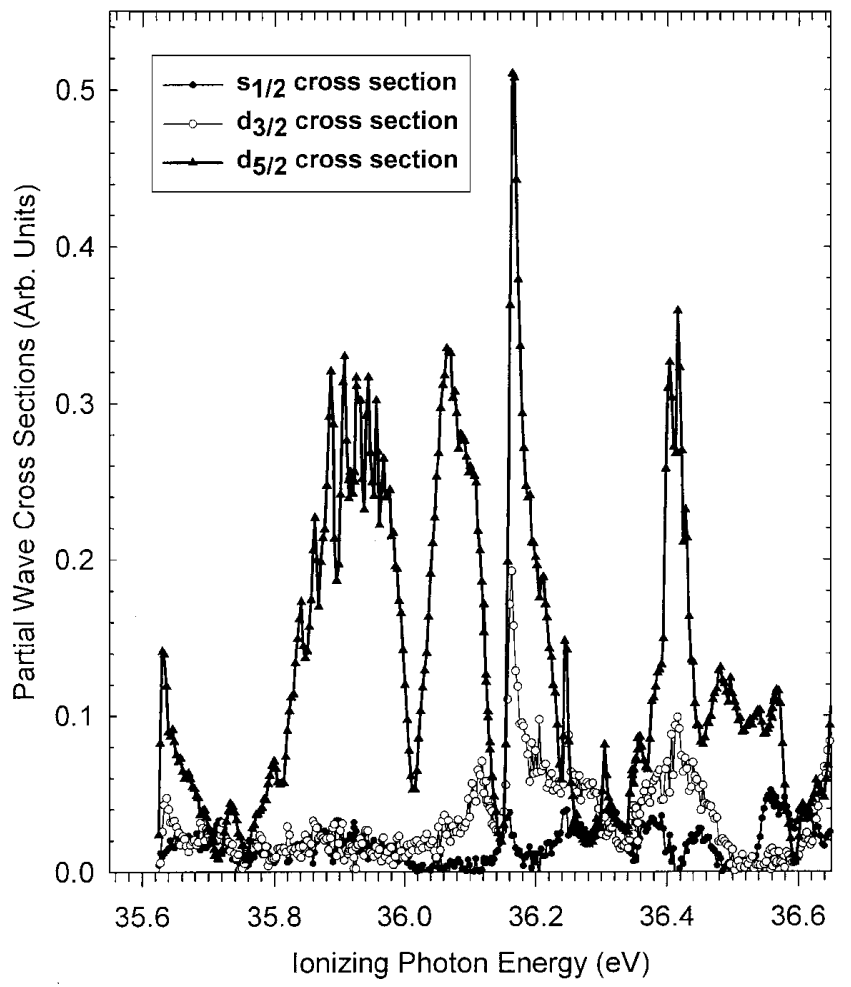

FIG. 3. Relative cross sections for $s_{1 / 2}$ (filled circles), $d_{3 / 2}$ (open circles), and $d_{5 / 2}$ (filled triangles) partial waves of the photoelectron. The lines are to guide the eye. 


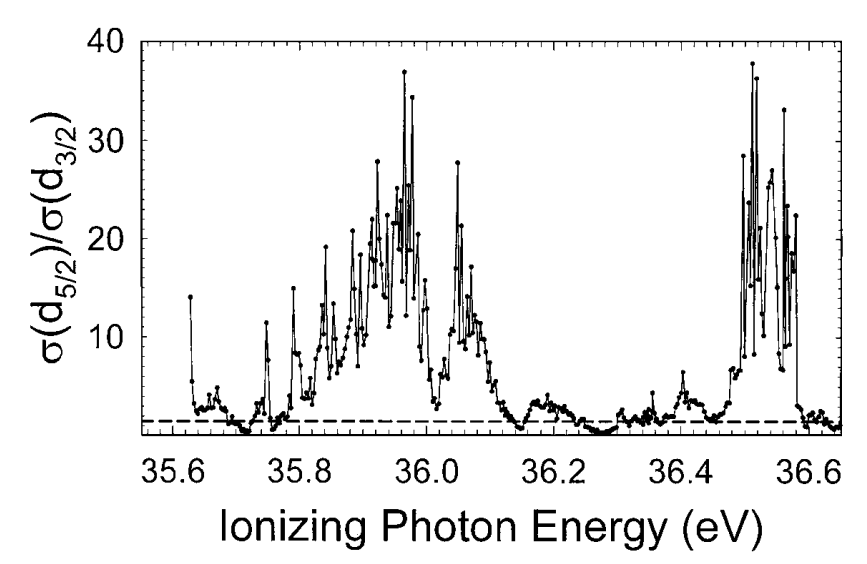

FIG. 4. The ratio of $d_{5 / 2}$ partial waves to $d_{3 / 2}$ partial waves. In the absence of relativistic interactions this ratio would have a constant value of $3 / 2$ (dashed line) as given by the multiplicities of the partial waves.

negligible, one would expect this ratio to have a constant value of 1.5 as given by the ratio of their multiplicities. Figure 4 shows dramatic deviations of the measured data from this value. While the tendency of the cross section ratio is to exceed the value of 1.5 , there are distinct regions (e.g., between 35.70 and $35.72 \mathrm{eV}$ and between 36.26 and $36.30 \mathrm{eV}$ ) where the opposite is true. The cause of this behavior is not clear to us; while the former energy range is characterized by what appears to be a strong ${ }^{2} F_{5 / 2} n f$ autoionizing resonance, there is no equivalent feature between 36.26 and $36.30 \mathrm{eV}$.

The analysis we have described provides new insight for the correlated photon-induced breakup of this system. At the same time, our analysis raises new questions. For example, is there a propensity rule for the total angular momentum $j_{e}$ of the photoelectron similar to the Fano propensity rule [24] that states $\ell+1$ partial waves are preferred over $\ell-1$ partial waves? Why does the $d_{5 / 2} / d_{3 / 2}$ partial wave cross section ratio become so large at certain energies? Why do certain autoionizing resonances have such a dominating effect on the ratio of the partial wave cross sections while others, even those in the same series, do not. It is hoped that further experiments and calculations will provide answers to these questions.

This work is supported by the National Science Foundation through Grants No. NSF-9731869 and No. 973520 (T. J. G.). We thank the entire staff of ALS for their dedicated work.

[1] V. Schmidt, Rep. Prog. Phys. 55, 1483 (1992).

[2] U. Becker and B. Langer, Phys. Scr. T78, 13 (1998).
[3] J. Jimenez-Mier, C. D. Caldwell, and D. L. Ederer, Phys. Rev. Lett. 57, 2260 (1986).

[4] K. W. McLaughlin, O. Yenen, and D. H. Jaecks, Phys. Rev. Lett. 81, 289 (1998).

[5] U. Heinzmann, F. Schafers, K. Thimm, A. Wolcke, and J. Kessler, J. Phys. B 12, L679 (1979).

[6] A. A. Wills, T. W. Gorczyca, N. Berrah, B. Langer, Z. Felfli, E. Kukk, J. D. Bozek, O. Nayandin, and M. Alshehri, Phys. Rev. Lett. 80, 5085 (1998).

[7] K. H. Schartner, B. Mobus, P. Lenz, B. Magel, H. Schmoranzer, and M. Wildberger, Phys. Scr. 41, 853 (1990); G. Mentzel, K. H. Schartner, O. Wilhelmi, B. Magel, U. Staude, F. Vollweiler, S. Lauer, H. Liebel, H. Schmoranzer, V.L. Sukhorukov, and B. M. Lagutin, J. Phys. B 31, 227 (1998).

[8] J. A. R. Samson, Y. Chung, and E. M. Lee, Phys. Rev. A 45, 259 (1992).

[9] H. W. van der Hart and C. H. Greene, J. Phys. B 32, 4029 (1999).

[10] U. Becker, R. Holzel, H. G. Kerkhoff, B. Langer, D. Szostak, and R. Wehlitz, Phys. Rev. Lett. 56, 1120 (1986).

[11] U. Heinzmann, J. Phys. B 13, 4353 (1980); 13, 4367 (1980).

[12] O. Yenen, K. W. McLaughlin, D. H. Jaecks, M. M. Sant'Anna, and E. A. Seddon, Phys. Rev. Lett. 86, 979 (2001).

[13] G. Snell, B. Langer, M. Drescher, N. Muller, B. Zimmermann, U. Hergenhahn, J. Viefhaus, U. Heinzmann, and U. Becker, Phys. Rev. Lett. 82, 2480 (1999).

[14] Z. Wang and D. S. Elliott, Phys. Rev. A 62, 053404 (2000); Phys. Rev. Lett. 84, 3795 (2000).

[15] O. Yenen, K. W. McLaughlin, and D. H. Jaecks, Phys. Rev. Lett. 79, 5222 (1997).

[16] O. Yenen, K. W. McLaughlin, D. H. Jaecks, and G. Snell, in Proceedings of the 22nd International Conference on the Physics of Electronic and Atomic Collisions, Abstracts of Contributed Papers, edited by S. Datz, M. E. Bannister, H. F. Krause, L. H. Saddiq, D. Schultz, and C. R. Vane (Rinton Press, Princeton, NJ, 2001), p. 675.

[17] U. Fano and J. H. Macek, Rev. Mod. Phys. 45, 553 (1973).

[18] Chris H. Greene and Richard N. Zare, Ann. Rev. Chem. 33, 119 (1982).

[19] R. P. Madden, D. L. Ederer, and K. Codling, Phys. Rev. 177, 136 (1969).

[20] L. Minnhagen, Ark. Fys. 25, 203 (1963); J. Opt. Soc. Am. 61, 1257 (1971).

[21] C. E. Moore, Atomic Energy Levels (U.S. GPO, Washington, D.C., 1971), Vol. I, pp. 211-218.

[22] A. A. Wills, A. A. Cafolla, F. J. Currell, J. Comer, A. Svensson, and M. A. MacDonald, J. Phys. B 22, 3217 (1989).

[23] J. A. R. Samson, E. M. Lee, and Y. Chung, Phys. Scr. 41, 850 (1990).

[24] U. Fano, Phys. Rev. 32, 617 (1985); see also C. H. Greene, in Fundamental Processes of Atomic Dynamics, edited by J. S. Briggs, H. Kleinpoppen, and H. O. Lutz (Plenum, New York, 1988), pp. 177-196. 